\title{
PERCEPCIÓN SOBRE LAS COMPETENCIAS GENERALES DE LOS DOCENTES DE ENFERMERÍA DEL ÁREA DE CUIDADOS INTENSIVOS, HOSPITAL GUILLERMO ALMENARA IRIGOYEN-2018
}

PERCEPTION ON THE GENERAL COMPETENCES OF THE NURSING TEACHERS OF THE INTENSIVE CARE AREA. HOSPITAL GUILLERMO ALMENARA IRIGOYEN, 2018

Carmen Susana Alva-Mayuri,a,b

\begin{abstract}
RESUMEN
Objetivo: Determinar la percepción sobre las competencias generales de los docentes de enfermería por enfermeros especialistas del área de cuidados intensivos en el Hospital Guillermo Almenara Irigoyen en el año 2018. Métodos: Estudio de tipo prospectivo y diseño con enfoque cuali-cuantitativo. Se encuestaron a 38 enfermeras mediante una escala de percepción de competencias generales, y se entrevistó a 7 enfermeras con una guía de entrevista, todas especialistas de cuidados intensivos. Se categorizó la percepción en buena, regular y mala. Se utilizó estadística descriptiva, mediante frecuencias absolutas

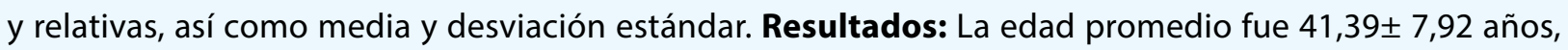
siendo el $92,1 \%$ de sexo femenino. Las competencias generales de los docentes de enfermería del área de cuidados intensivos alcanzaron una percepción regular en $55,3 \%$ y buena en $44,7 \%$. Los aspectos con mayor grado de acuerdo fueron adaptación a las diferentes situaciones que se presentan en la práctica diaria $(65,8 \%)$, la empatía mostrada a los estudiantes $(57,9 \%)$ y la capacidad para resolver problemas de emergencia (50\%). Los años de experiencia fue lo más relevante referido a la adecuada capacidad y ejecución de las enfermeras. Conclusión: La percepción sobre las competencias generales de los docentes de enfermería del área de cuidados intensivos del Hospital Guillermo Almenara Irigoyen en el año 2018 fue regular.
\end{abstract}

Palabras clave: Percepción; Competencias generales; Enfermera; Cuidados intensivos. (fuente: DeCS BIREME)

\begin{abstract}
Objective: To determine the perception on the general competences of nursing teachers by specialist nurses in the intensive care area at the Guillermo Almenara Irigoyen Hospital in 2018. Key words: general competences, nurse, intensive care. Methods: Prospective study and design with qualitative-quantitative approach. Thirty-eight nurses were surveyed using a general skills perception scale, and 7 nurses were interviewed with an interview guide, all intensive care specialists. The perception was categorized into good, fair and bad. Descriptive statistics was used, using absolute and relative frequencies, as well as mean and standard deviation. Results: The average age was $41.39 \pm 7.92$ years, $92.1 \%$ being female. The general competencies of nursing teachers in the intensive care area reached a regular perception of $55.3 \%$ and good perception in $44.7 \%$. The aspects with the highest degree of agreement were adaptation to the different situations that occur in daily practice $(65.8 \%)$, the empathy shown to students (57.9\%) and the ability to solve emergency problems (50\%). The years of experience were the most relevant referred to the adequate capacity and execution of the nurses. Conclusion: The perception about the general competences of the nursing teachers of the intensive care area of the Guillermo Almenara Irigoyen Hospital in the year 2018 was regular.
\end{abstract}

Key words: Perception; General skills; Nurse; Intensive care. (source: MeSH NLM)

\footnotetext{
${ }^{1}$ Hospital Guillermo Almenara Irigoyen, Lima - Perú.

a Licenciada en enfermería.

${ }^{\mathrm{b}}$ Especialista en Cuidados Intensivos
}

Citar como: Carmen Susana Alva-Mayuri. Percepción sobre las competencias generales de los docentes de enfermería del área de cuidados intensivos. Hospital Guillermo Almenara Irigoyen, 2018. Rev. Fac. Med. Hum. Julio 2019; 19(3):69-74. DOI 10.25176/RFMH.v19i3.2159 


\section{INTRODUCCIÓN}

Uno de los modelos educativos que reúnen los enfoques vigentes de las leyes educativas es el de las competencias. No hay manuscrito, ni reforma que no envuelva como mecanismo indispensable la orientación educativa por competencias' ${ }^{1}$. El Proyecto Tuning, uno de los pioneros, cuya meta es prestar la óptima unificación de las sapiencias con las acciones laborales en las instituciones, a partir de un cambio de cultura en el planteamiento de las propuestas formativas en la educación².

Las competencias son aquellas peculiaridades de un individuo que están afines con el desempeño positivo de un trabajo y pueden ser frecuentes en otras circunstancias ${ }^{3}$. Por otro lado, las competencias genéricas implican una serie de rasgos en la esfera cognitiva y motivacional, que se manifiestan a través de las competencias instrumentales como capacidad de análisis, síntesis, de organización, planificación, y de gestión de información; de las competencias personales como el hecho de laborar en equipo, la destreza para la conducción de las relaciones con los demás y la responsabilidad ética; y de las competencias sistémicas como aprendizaje autónomo, ajuste a nuevas condiciones, creatividad y liderazgo, entre otras ${ }^{4}$.

Las competencias del profesional de enfermería, como los docentes, no solo se apoyan en saberes o destrezas fraccionadas, sino más bien se edifican en función de la sucesión de acciones de aprendizaje, las cuales giran en torno a la relevancia y trascendencia que se da al trabajador, siendo su capacidad, su razón, y su aporte de ideas la que logra trascendencia para el ajuste de las variaciones, con la finalidad de perfeccionar la atención y desarrollo del profesional de enfermería ${ }^{5}$. Dicho profesional, al estar trabajando en un área de especial cuidado, debe proporcionar una adecuada atención a los pacientes y formación educativa a sus alumnos, pues estos últimos serán los futuros especialistas que realizarán importantes funciones en el área de Cuidados Intensivos. Sin embargo, algunas veces se observa una deficiente labor en aspectos básicos que debería manejar, quizá por la poca orientación, tanto teórica como práctica.

Cabe resaltar, que en un trabajo desarrollado por Argüello (2016) se encontró que las principales competencias evidenciadas en el tutor de práctica de enfermería fueron el saber disciplinar actualizado, adecuado desenvolvimiento docente, buena

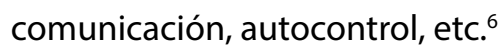

Teniendo en cuenta lo mencionado, el presente trabajo, brinda datos relevantes sobre el perfil de competencias generales de los docentes de enfermería de la Unidad de Cuidados Intensivos, en base a las percepciones de sus colegas especialistas que no laboran como docentes, quienes contribuyeron a revelar las características de sus funciones y habilidades tras observarlos al momento de su desempeño, además, esto sirve como un precedente que motive al desarrollo de otras investigaciones, ya que hay poco material enfocado a este tema de estudio.

El objetivo de este artículo es determinar la percepción sobre las competencias generales de los docentes de enfermería por enfermeros especialistas del área de cuidados intensivos en el Hospital Guillermo Almenara Irigoyen en el año 2018.

\section{MÉTODOS}

Se realizó estudio de tipo prospectivo y de diseño con enfoque cuali-cuantitativo. La muestra de estudio estuvo conformada por 38 enfermeras para la fase cuantitativa, y 7 enfermeras para la fase cualitativa, todas con título de especialista que laboran en la Unidad de Cuidados Intensivos del Hospital Guillermo Almenara Irigoyen durante el año 2018, que cumplieron los criterios de inclusión y exclusión.

\section{Criterios de inclusión:}

- Enfermeras especialistas del área de cuidados intensivos del hospital Guillermo Almenara Irigoyen.

- Enfermeras especialistas con un mínimo de 5 años de experiencia laborando en el área de cuidados intensivos.

- Enfermeras especialistas que no se dedican a la docencia práctica.

\section{Criterios de exclusión:}

- Enfermeras especialistas que no son del área de cuidados intensivos.

- Enfermeras especialistas en cuidados intensivos que laboran menos de 5 años en el área en mención.

- Enfermeras especialistas que no están de acuerdo en participar en el trabajo de investigación y no firmen el consentimiento informado.

La técnica utilizada fue la entrevista y encuesta. Se usaron dos instrumentos: Una Guía de entrevista (Fase cualitativa), conformada por 2 preguntas básicas sobre la percepción de las enfermeras especialistas acerca de la forma de enseñanza práctica de sus colegas. El otro instrumento fue la escala de percepción sobre competencias generales (Fase cuantitativa), conformada por 14 ítems, con respuesta tipo Likert con 5 alternativas de respuesta donde: 
- Totalmente de acuerdo equivale a 5

- De acuerdo equivale a 4

- Ni de acuerdo y en desacuerdo equivale a 3

- En desacuerdo equivale a 2

- Totalmente en desacuerdo equivale a 1

Ambos instrumentos fueron validados mediante juicio de expertos a través de la prueba binomial, obteniendo concordancia entre sus apreciaciones $(p<0,05)$. Asimismo, la escala fue confiabilizada por una prueba piloto, obteniendo un coeficiente de alfa de Cronbach de 0,91 , es decir una muy alta confiabilidad, siendo el instrumento apto para su aplicación.

Para categorizar la percepción de las competencias generales, se obtuvo una calificación total de 14 a 70 puntos, y realizó una baremación de la siguiente manera:

$\mathrm{K}=\left(\mathrm{N}^{\circ}\right.$ de ítems $\mathrm{x}$ puntaje mayor $)-\mathrm{N}^{\circ}$ de ítems $) \div 3$

$K=(14 \times 5)-14) \div 3=18$

Con el valor de la constante $(K)$ se utiliza para obtener las siguientes categorías:

Percepción buena: 52 - 70 puntos.

Percepción regular: 33 - 51 puntos.

Percepción mala: 14 - 32 puntos.

Para la medición de la variable se usó la estadística descriptiva, la frecuencia absoluta, porcentajes y promedio aritmético. Los resultados se presentaron en gráficos y/o tablas estadísticas. Para la fase cualitativa: se degrabaron las entrevistas realizadas.

Se ejecutó el estudio con la aprobación del Comité Institucional de Ética en Investigación (CIEI) del Hospital Guillermo Almenara Irigoyen, y se cumplió los principios bioéticos, aplicando el respectivo consentimiento informado a las enfermeras de cuidados intensivos, protegiendo la confidencialidad de sus datos.

\section{RESULTADOS}

Se encuestó a un total de 38 enfermeros con título de especialista que laboran en la Unidad de Cuidados Intensivos del Hospital Guillermo Almenara Irigoyen durante el año 2018. La edad promedio fue 41,39 $\pm 7,92$ años, siendo el $92,1 \%$ fue de sexo femenino y $7,9 \%$ masculino (Figura 1)

Las competencias generales de los docentes de enfermería del área de cuidados intensivos alcanzaron una percepción regular en 55,3\% y buena en $44,7 \%$. (Figura 2).
En la tabla 1 se observa uno de los aspectos evaluados dentro de las competencias generales de docentes fue respecto a su adaptación a las diferentes situaciones que se presentan en la práctica diaria, el cual recibió un $65,8 \%$ de acuerdo, y la capacidad para resolver problemas de emergencia con un 50\% de acuerdo. Para complementar estos puntos, se les entrevistó respecto a la ejecución adecuada los procedimientos dirigidos al paciente, manifestando que "..la enfermera intensivista tiene ya en la memoria todos los procedimientos, no es necesario correr a coger la guía de procedimientos para saber que materiales o cómo va a ser el procedimiento, uno ya por la experiencia y por los años ya sabe que materiales..", así mismo también mencionaron que "... en la gran mayoría ejecutan sus procedimientos de acuerdo a los protocolos que manejamos y hay años de experiencia, una que otra que de repente pueda que se confíen y pueden obviar algunos pasos de los protocolos largos pero en líneas generales ejecutan de acuerdo a los procedimientos según los protocolos establecidos", por otro lado, también declaran algo negativo "... me parece que estamos fallando bastante en lo que es bioseguridad".

Un aspecto también evaluado dentro de las competencias, es la empatía mostrada a los estudiantes que fue respondido como de acuerdo por un 57,9\%, sin embargo, el que tomen en cuenta la opinión del estudiante se respondió indiferente por un 39,5\%. (Tabla 1). Al momento de entrevistar a las enfermeras respecto a la interrelación entre el docente y alumno, manifestaron "... hay una relación cordial manteniendo una línea de autoridad de docente a alumno", así mismo "es una interrelación directa ya que las prácticas se hacen en las horas de trabajo de tal manera que el alumno no solo ve los diferentes procedimientos sino tiene la facilidad de preguntar porque se pueden realizar dichos procedimientos y bajo qué circunstancias".

Por otro lado, los aspectos considerados como respuestas en desacuerdo, fueron que el docente organiza y distribuye su tiempo para ejercer su labor en la práctica intensivista y docente en un $26,3 \%$, y el docente trabaja en equipo con los demás colegas en un 21,1\%. (Tabla 1) Respecto a esto último, respondieron que "el docente de practica de $\mathrm{UCl}$, generalmente domina algunas áreas de la especialidad más que otras, y generalmente no pide a sus colegas que dominan otras áreas para que la apoyen en la formación de esos temas o procedimientos que no dominan". 
Por último, el uso de Tecnologías de la Información y la Comunicación (TICS) por parte del docente se mostró como de acuerdo en un 55,3\%, pero la actualización de sus conocimientos a través de capacitaciones de manera constante fue respondida de manera indiferente en $47,4 \%$, $y$ el liderazgo del docente en $39.5 \%$ en desacuerdo. (Tabla 1).

Tabla 1. Ítems de competencias generales de docentes de enfermería del área de cuidados intensivos del Hospital Guillermo Almenara Irigoyen en el año 2018.

\begin{tabular}{|c|c|c|c|c|c|c|c|c|c|c|}
\hline \multirow[t]{2}{*}{ Ítems sobre competencias generales } & \multicolumn{2}{|c|}{$\begin{array}{l}\text { Totalmente de } \\
\text { acuerdo }\end{array}$} & \multicolumn{2}{|c|}{ De acuerdo } & \multicolumn{2}{|c|}{$\begin{array}{c}\text { Ni de } \\
\text { acuerdo, ni en } \\
\text { desacuerdo }\end{array}$} & \multicolumn{2}{|c|}{$\begin{array}{l}\text { En } \\
\text { desacuerdo }\end{array}$} & \multicolumn{2}{|c|}{$\begin{array}{l}\text { Totalmente en } \\
\text { desacuerdo }\end{array}$} \\
\hline & $\mathbf{N}$ & $\%$ & $\mathbf{N}$ & $\%$ & $\mathbf{N}$ & $\%$ & $\mathbf{N}$ & $\%$ & $\mathbf{N}$ & $\%$ \\
\hline $\begin{array}{l}\text { El docente hace uso de las Tecnología de } \\
\text { Información y comunicación (TICs). }\end{array}$ & 7 & $18.4 \%$ & 21 & $55.3 \%$ & 7 & $18.4 \%$ & 3 & $7.9 \%$ & 0 & $0.0 \%$ \\
\hline $\begin{array}{l}\text { El docente aplica sus conocimientos en la práctica } \\
\text { intensivista. }\end{array}$ & 10 & $26.3 \%$ & 21 & $55.3 \%$ & 7 & $18.4 \%$ & 0 & $0.0 \%$ & 0 & $0.0 \%$ \\
\hline $\begin{array}{l}\text { El docente actualiza sus conocimientos recibiendo } \\
\text { capacitaciones de manera constante. }\end{array}$ & 4 & $10.5 \%$ & 13 & $34.2 \%$ & 18 & $47.4 \%$ & 3 & $7.9 \%$ & 0 & $0.0 \%$ \\
\hline $\begin{array}{l}\text { El docente tiene una buena comunicación con el } \\
\text { estudiante, creando un clima de confianza. }\end{array}$ & 9 & $23.7 \%$ & 13 & $34.2 \%$ & 13 & $34.2 \%$ & 3 & $7.9 \%$ & 0 & $0.0 \%$ \\
\hline $\begin{array}{l}\text { El docente toma decisiones adecuadas con el equipo } \\
\text { multidisciplinario ante una urgencia. }\end{array}$ & 9 & $23.7 \%$ & 15 & $39.5 \%$ & 8 & $21.1 \%$ & 5 & $13.2 \%$ & 1 & $2.6 \%$ \\
\hline El docente trabaja en equipo con los demás colegas. & 2 & $5.3 \%$ & 18 & $47.4 \%$ & 10 & $26.3 \%$ & 8 & $21.1 \%$ & 0 & $0.0 \%$ \\
\hline El docente toma en cuenta la opinión del estudiante. & 5 & $13.2 \%$ & 12 & $31.6 \%$ & 15 & $39.5 \%$ & 6 & $15.8 \%$ & 0 & $0.0 \%$ \\
\hline El docente mantiene un comportamiento ético. & 6 & $15.8 \%$ & 19 & $50.0 \%$ & 8 & $21.1 \%$ & 5 & $13.2 \%$ & 0 & $0.0 \%$ \\
\hline El docente muestra empatía con los estudiantes. & 9 & $23.7 \%$ & 22 & $57.9 \%$ & 7 & $18.4 \%$ & 0 & $0.0 \%$ & 0 & $0.0 \%$ \\
\hline $\begin{array}{l}\text { El docente se interesa por los (las) estudiantes que } \\
\text { muestran dificultades en el aprendizaje. }\end{array}$ & 7 & $18.4 \%$ & 15 & $39.5 \%$ & 10 & $26.3 \%$ & 5 & $13.2 \%$ & 1 & $2.6 \%$ \\
\hline $\begin{array}{l}\text { El docente tiene la capacidad para resolver } \\
\text { problemas de emergencia. }\end{array}$ & 12 & $31.6 \%$ & 19 & $50.0 \%$ & 6 & $15.8 \%$ & 1 & $2.6 \%$ & 0 & $0.0 \%$ \\
\hline El docente ejerce liderazgo. & 11 & $28.9 \%$ & 12 & $31.6 \%$ & 15 & $39.5 \%$ & 0 & $0.0 \%$ & 0 & $0.0 \%$ \\
\hline $\begin{array}{l}\text { El docente se adapta a las diferentes situaciones que } \\
\text { se le presentan en su práctica diaria. }\end{array}$ & 6 & $15.8 \%$ & 25 & $65.8 \%$ & 7 & $18.4 \%$ & 0 & $0.0 \%$ & 0 & $0.0 \%$ \\
\hline $\begin{array}{l}\text { El docente organiza y distribuye su tiempo para } \\
\text { ejercer su labor en la práctica intensivista y docente. }\end{array}$ & 5 & $13.2 \%$ & 13 & $34.2 \%$ & 7 & $18.4 \%$ & 10 & $26.3 \%$ & 3 & $7.9 \%$ \\
\hline
\end{tabular}

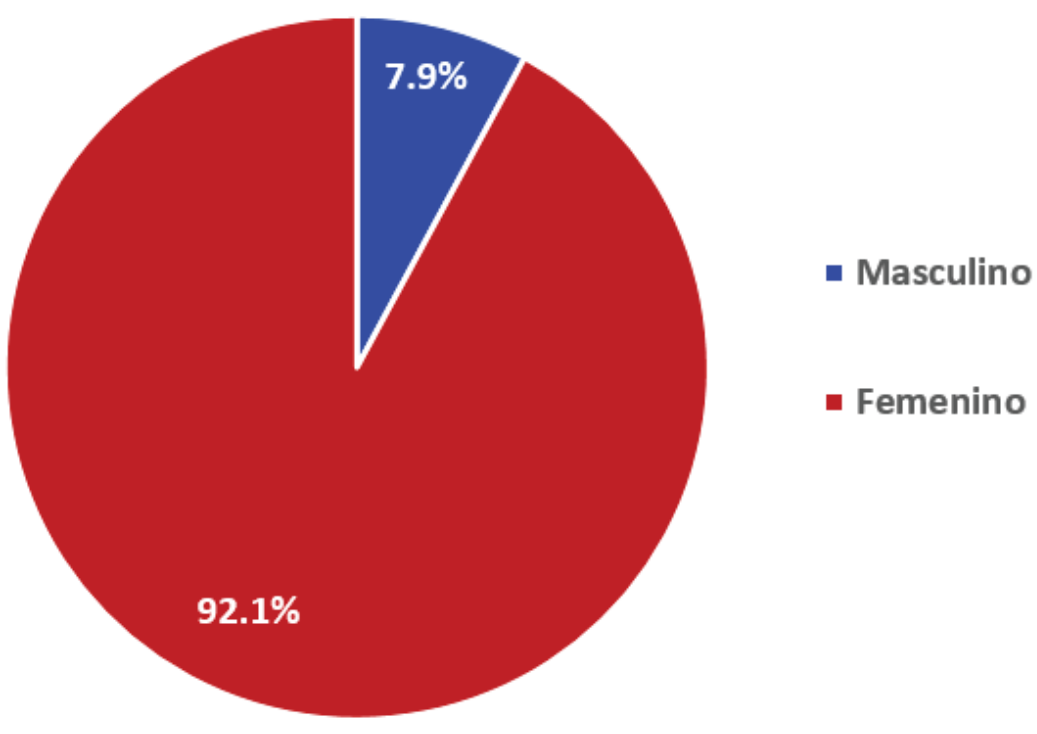

Figura 1. Sexo de enfermeros especialistas del Hospital Guillermo Almenara Irigoyen durante el año 2018. 


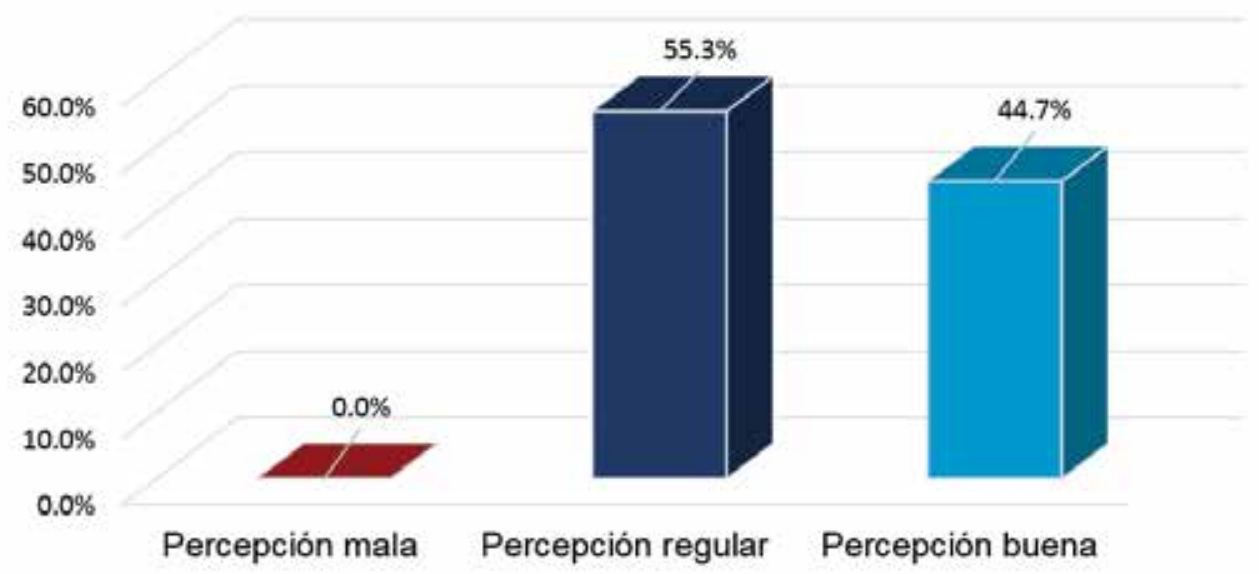

Figura 2. Percepción de competencias generales de docentes de enfermería del área de cuidados intensivos del Hospital Guillermo Almenara Irigoyen en el año 2018.

\section{DISCUSIÓN}

El docente de práctica clínica, en este caso de enfermería, cumple un rol de gran importancia y de implicancia en el estudiante, y va más allá de la enseñanza de procedimientos y técnicas, sino que es un acompañamiento continuo y de compartir experiencias, todo ello tendrá un efecto en el desarrollo de los futuros profesionales. Por ello los docentes deben tener sus competencias adecuadas.

En el presenteestudiolapercepción delascompetencias generales de los docentes de enfermería de práctica clínica en cuidados intensivos fue principalmente de regular a bueno. En este punto, coincide con el estudio que encontró que el $75,72 \%$ de estudiantes perciben las competencias en proceso de desarrollo, $y$ $20,23 \%$ lo perciben como competencias desarrolladas ${ }^{7}$, diferente a lo evidenciado en otra investigación donde encontraron que la percepción general de los estudiantes respecto al rol de los profesores en la formación del cuidado humanizado es considerada Buena en un $98 \%{ }^{8}$, así también otra investigación describe que la percepción del desempeño docente en la práctica clínica por parte de los alumnos fue muy buena en un $69 \%{ }^{9}$.

Las competencias referidas a la adaptación a las diferentes situaciones en la práctica diaria, así como la capacidad de resolver problemas de emergencia por parte de los docentes fueron aceptadas por la mayoría de las enfermeras encuestadas. Muchos de estos aspectos son avalados, según refieren, por los años de experiencia que tienen las enfermeras docentes, lo cual hace que el manejo de la práctica sea muy pertinente basándose en protocolos y normativas. Sin embargo, es importante recalcar que, a pesar de ello, hay cuestiones que debilitan su actuar clínico como es la bioseguridad. Respecto a esto una investigación refiere que entre las competencias imprescindibles se destacaron el conocimiento disciplinar actualizado $(87,59 \%)$, y la capacidad docente $(49,16 \%)^{6}$.

Otro aspecto determinado en el estudio es la empatía mostrada a los estudiantes, así como tomar en cuenta su opinión, lo cual fue percibido de manera favorable por la mayoría, destacando la cordialidad con la que se interrelacionaba el docente con el estudiante, así como la oportunidad de preguntar u opinar en diferentes procesos durante el desarrollo de la práctica; en este sentido, otros autores, en su trabajo califican la percepción que tienen los estudiantes de enfermería en la interacción docente - estudiantes como buena en el $94 \%$ y solo $6 \%$ es regular ${ }^{8}$. Es necesario considerar que la relación que el docente establezca con el estudiante, podría ser determinante en el desarrollo del proceso de aprendizaje, pues generará un mejor clima y con ello un mejor desenvolvimiento en ambas partes.

\section{CONCLUSIÓN}

En base a los resultados obtenidos en esta investigación se concluye que la percepción sobre las competencias generales de los docentes de enfermería del área de cuidados intensivos del Hospital Guillermo Almenara Irigoyen en el año 2018 fue principalmente regular. 
Contribuciones de autoría: La autora participó en la génesis de la idea, diseño de proyecto, recolección e interpretación de datos, análisis de resultados y preparación del manuscrito del presente trabajo de investigación.

Financiamiento: Autofinanciado.

Correspondencia: Carmen Susana Alva Mayuri.

Dirección: Jr. Máximo Alvarado Nro 286 - San Juan de Miraflores, Lima-Perú. Teléfono: 995039075

Correo:xuperxu9@gmail.com
Conflicto de interés: La autora declara no tener conflicto de interés en la publicación de este artículo.

Recibido: 15 de abril 2019

Aprobado: 30 de junio 2019

\section{REFERENCIAS BIBLIOGRÁFICAS}

1.Alonso C, Gallego D. Los estilos de aprendizaje como competencias para el estudio, el trabajo y la vida. Revista Estilos de Aprendizaje [En Línea]. 2010 [Acceso el 08 de febrero del 2018]; 6(6). Disponible en:https://www2.uned.es/revistaestilosdeaprendizaje/numero_6/ articulos/lsr_6_articulo_1.pdf

2.Ramos J. Formación por competencias en el SSPA. España: Hospital de Jerez del Servicio Andaluz de Salud; 2018. [Acceso el 19 de febrero del 2018]. Disponible en: https://rodin.uca.es/xmlui/ bitstream/handle/10498/20564/FORMACI\%C3\%93N\%20POR\%20 COMPETENCIAS\%20EN\%20EL\%20SSPA.pdf?sequence=1\&isAllowed=y

3.Mulder M, Weigel T, Collings K. El concepto de competencia en el desarrollo de la educación y formación profesional en algunos Estados miembros de la UE: un análisis crítico. Profesorado. Revista de Currículum y Formación de Profesorado [En Línea]. 2008 [Acceso el 22 de marzo del 2018]; 12(3): 1-25. Disponible en: http://www.redalyc.org/ pdf/567/56712875007.pdf

4.González V, González R. Competencias genéricas y formación profesional: un análisis desde la docencia universitaria. Revista Iberoamericana de Educación [En Línea]. 2008 [Acceso el 05 de abril del 2018]; 47: 185-209. Disponible en: https://rieoei.org/RIE/article/ view/710

5.González C. Competencias profesionales en enfermeras que desempeñan su labor en una Unidad de cuidados Intensivos. Revista Iberoamericana de Educación e Investigación en Enfermería [En Línea].
2015 [Acceso el 09 de abril del 2018]; 5(1): 35-46. Disponible en: https:// www.enfermeria21.com/revistas/aladefe/articulo/149/competenciasprofesionales-en-enfermeras-que-desempenan-su-labor-en-unaunidad-de-cuidados-intensivos/

6.Argüello M. Desarrollo del Perfil de Competencias del Tutor de las Prácticas Clínicas de Enfermería. [Tesis doctoral]. Madrid: Universidad Autónoma de Madrid; 2016. [Acceso el 09 de mayo del 2018]. Disponible en: https://repositorio.uam.es/handle/10486/678307

7.Labajos N. Competencias de los docentes de Enfermería de la UNMSM según percepción de los estudiantes 2013. [Tesis de Licenciatura]. Lima-Perú: Universidad Nacional Mayor de San Marcos, Facultad de Medicina; 2014. [Acceso el 10 de mayo del 2018]. Disponible en: http:// cybertesis.unmsm.edu.pe/bitstream/handle/cybertesis/3533/Labajos_ an.pdf? sequence $=1$ \&isAllowed $=y$

8.Acevedo J, Alvarado L. Percepción de los estudiantes de la Escuela de Enfermería Padre Luis Tezza acerca del rol docente en la formación del cuidado humanizado. [Tesis de Licenciatura]. Lima-Perú: Universidad Ricardo Palma; 2015. [Acceso el 21 de mayo del 2018]. Disponible en:http://cybertesis.urp.edu.pe/bitstream/urp/391/1/Acevedo_ jc\%3B\%2BAlvarado_lc.pdf

9.Uribe L. Percepción de los Alumnos de Enfermería sobre el Desempeño docente en la Práctica Clínica. Revista Enfermería Universitaria ENEOUNAM [En Línea]. 2010 [Acceso el 28 de mayo del 2018]; 7(4): 29-34 Disponible en: http://www.scielo.org.mx/pdf/eu/v7n4/v7n4a5.pdf

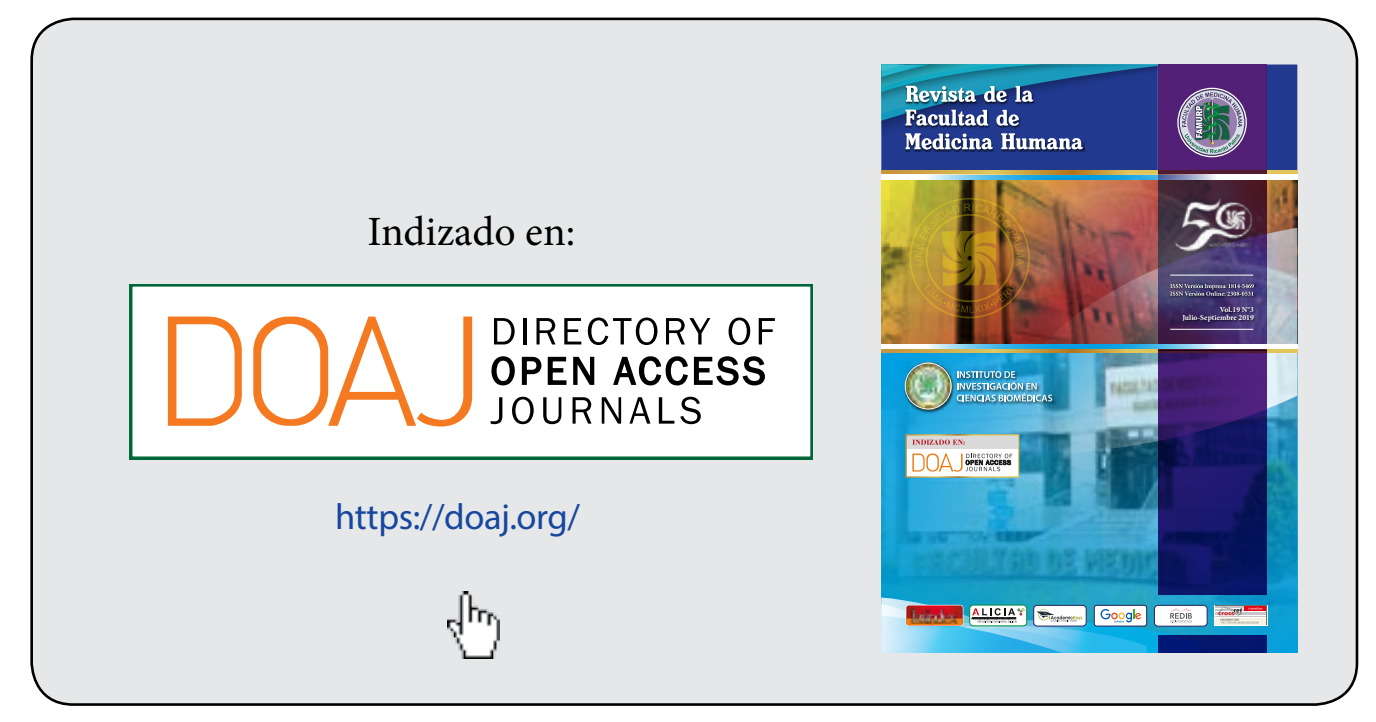

